\title{
Measurements of the $\mathrm{OH}$ radical yield from the ozonolysis of biogenic alkenes: A potential interference with laser-induced fluorescence measurements of ambient $\mathrm{OH}$
}

Pamela Rickly ${ }^{1}$ and Philip S. Stevens ${ }^{1,2}$

${ }^{1}$ School of Public and Environmental Affairs, Indiana, University, Bloomington, IN USA

${ }^{2}$ Department of Chemistry, Indiana University, Bloomington, IN USA

Correspondence to: Philip S. Stevens (pstevens@indiana.edu)

Supplementary Information
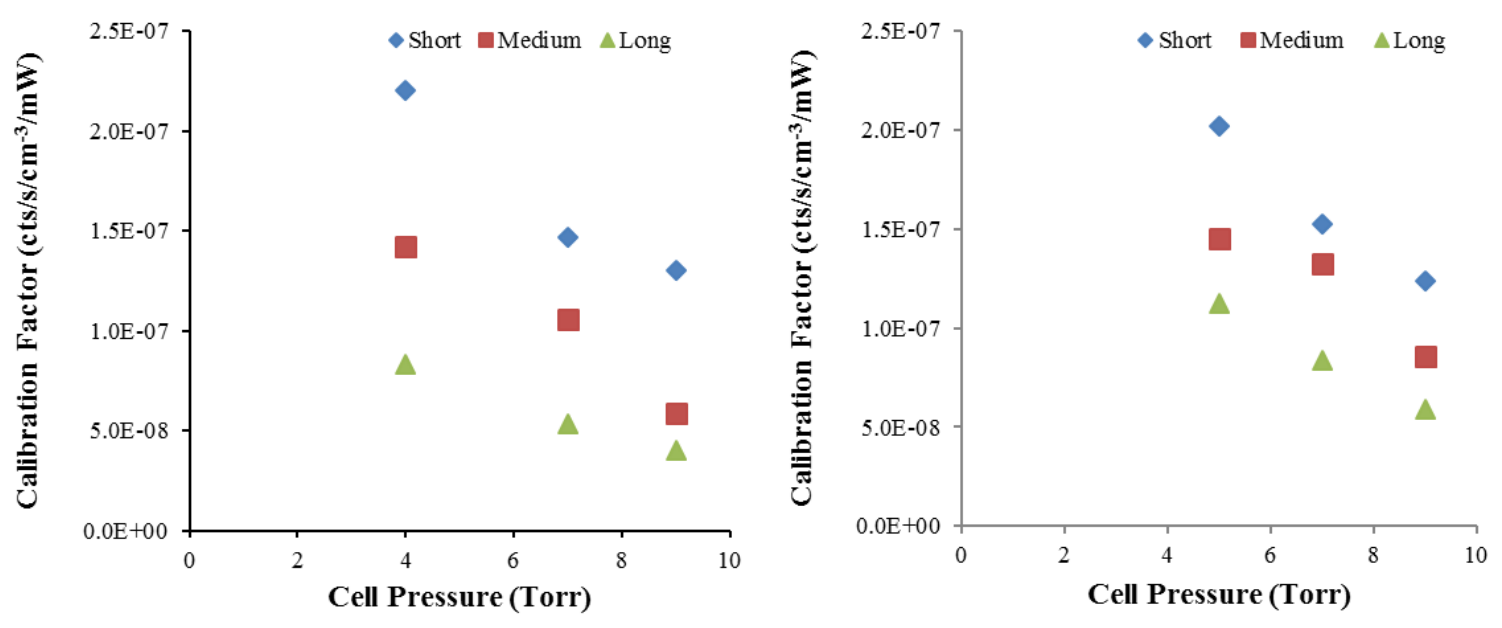

Figure S1. Calibration factors based on inlet length and diameter for the $1 \mathrm{~mm}$ diameter inlet (left) and the $0.6 \mathrm{~mm}$ diameter inlet (right).
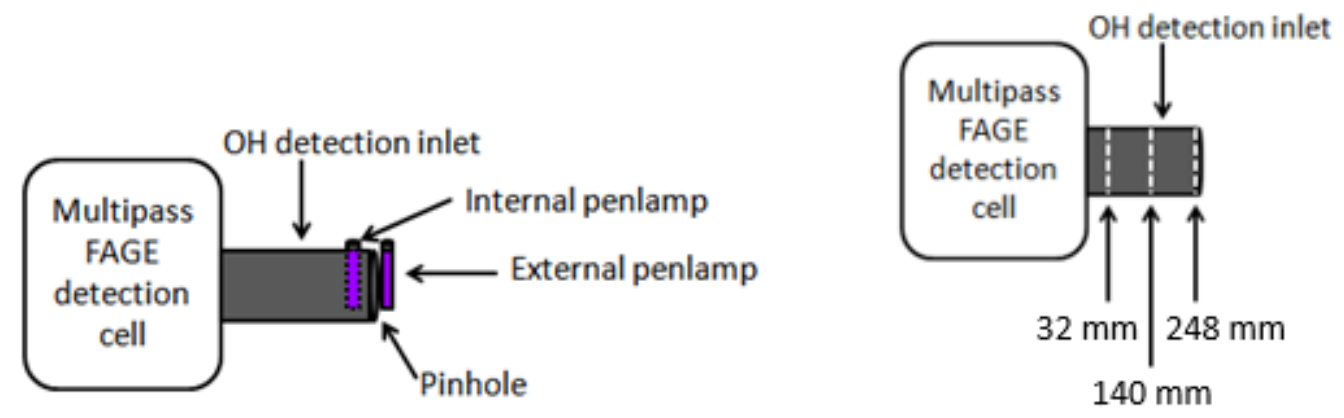

Figure S2. Placement of mercury penlamps to determine the efficiency of the external $\mathrm{OH}$ titration (left) and the varying inlet lengths used in the experiments (right). 

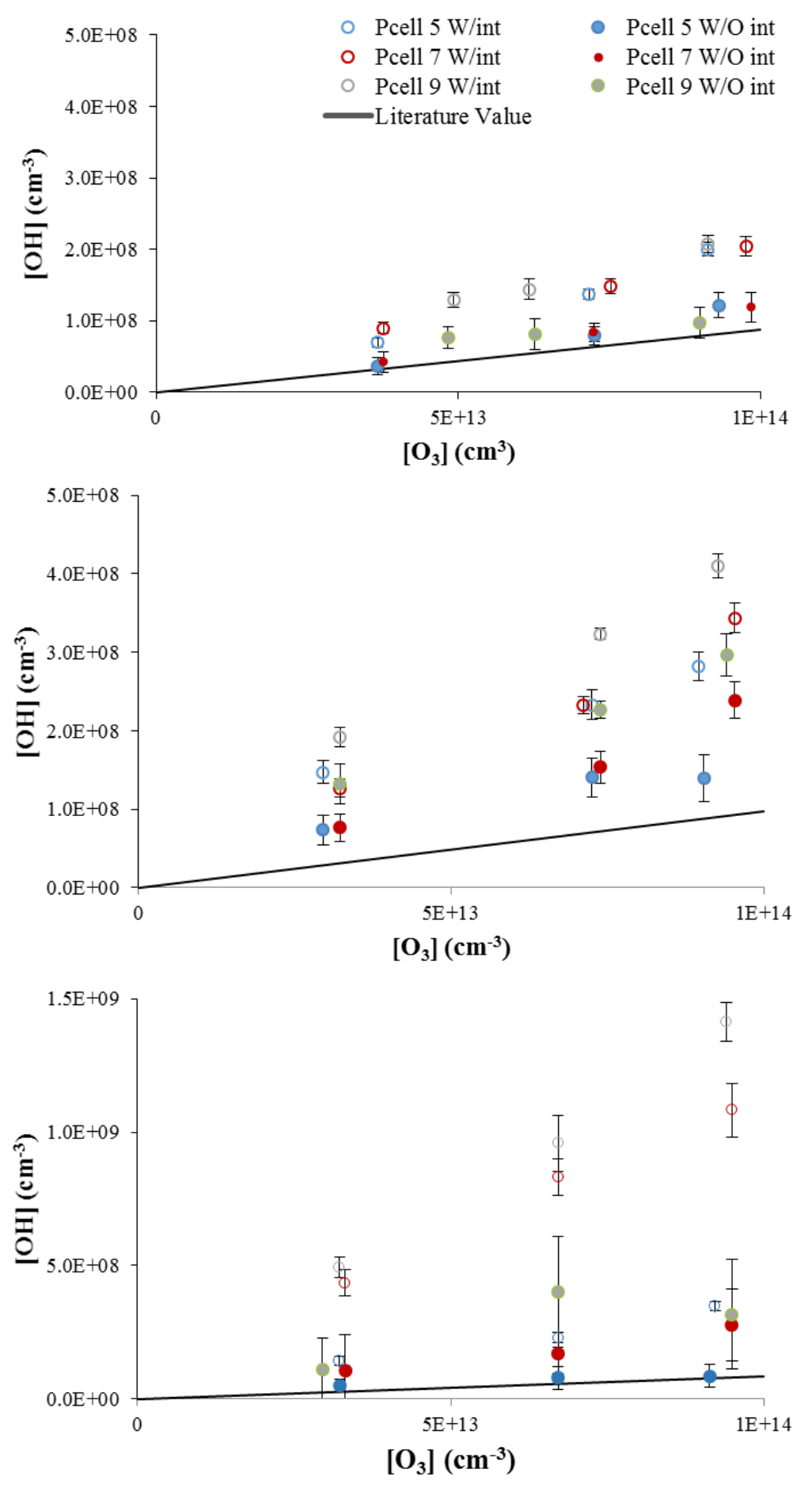

Figure S3. OH concentrations from $\alpha$-pinene ozonolysis at 3 cell pressures with and without the interference using the $1 \mathrm{~mm}$ inlet and the short (top), medium (middle), and long (bottom) inlet lengths. Error bars indicate the precision of the measurement $(1 \sigma)$. 

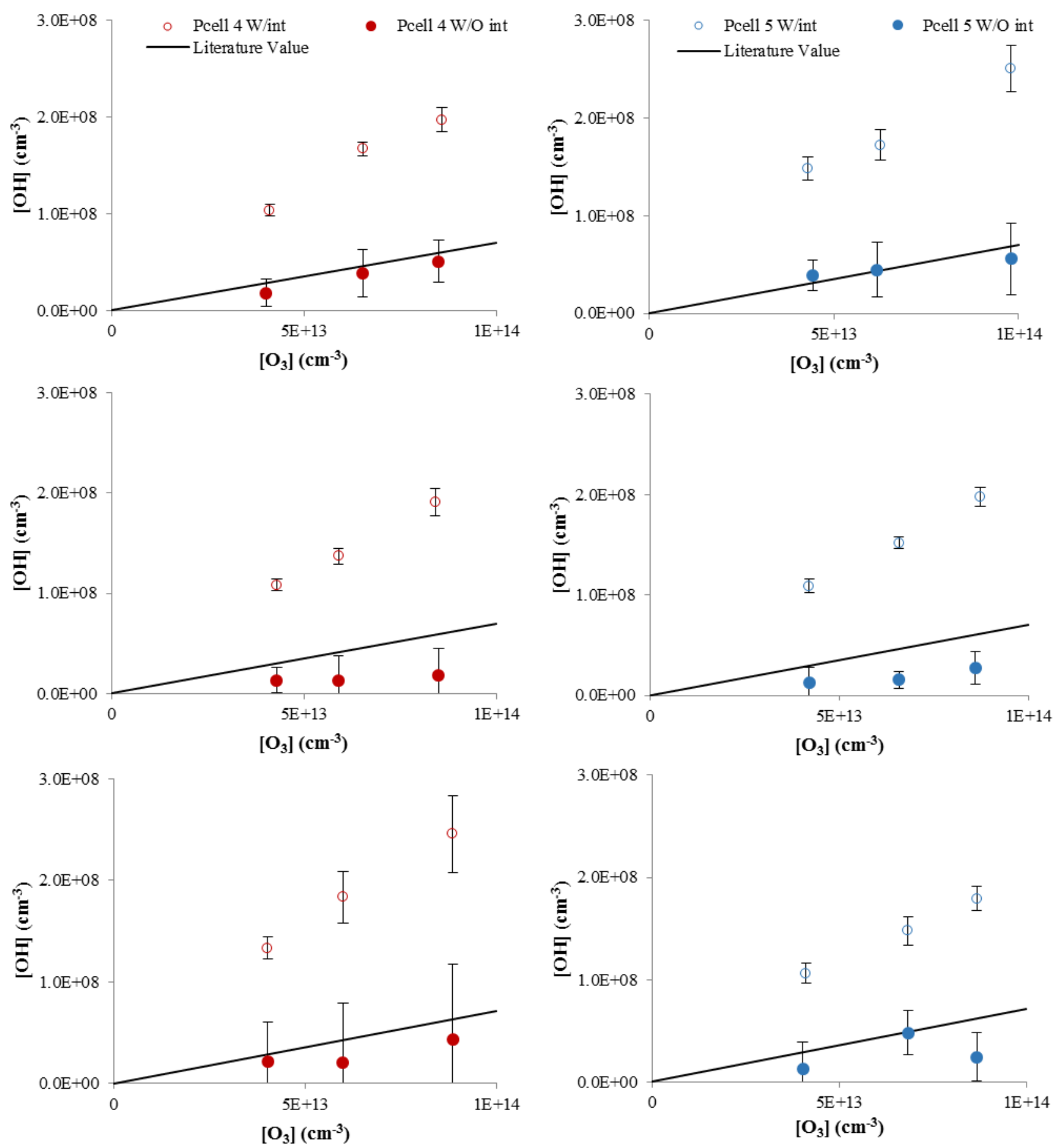

Figure S4. OH concentrations from ocimene ozonolysis with an ocimene concentration of $1 \times 10^{13}$ molecules cm${ }^{-3}$. The left column shows the results using the $0.6 \mathrm{~mm}$ inlet diameter and the right column using the $1 \mathrm{~mm}$ inlet diameter. The top row shows results from the short inlet length, middle row from the mid-length inlet, and the last row from the long inlet length. Error bars indicate the precision of the measurement $(1 \sigma)$. 

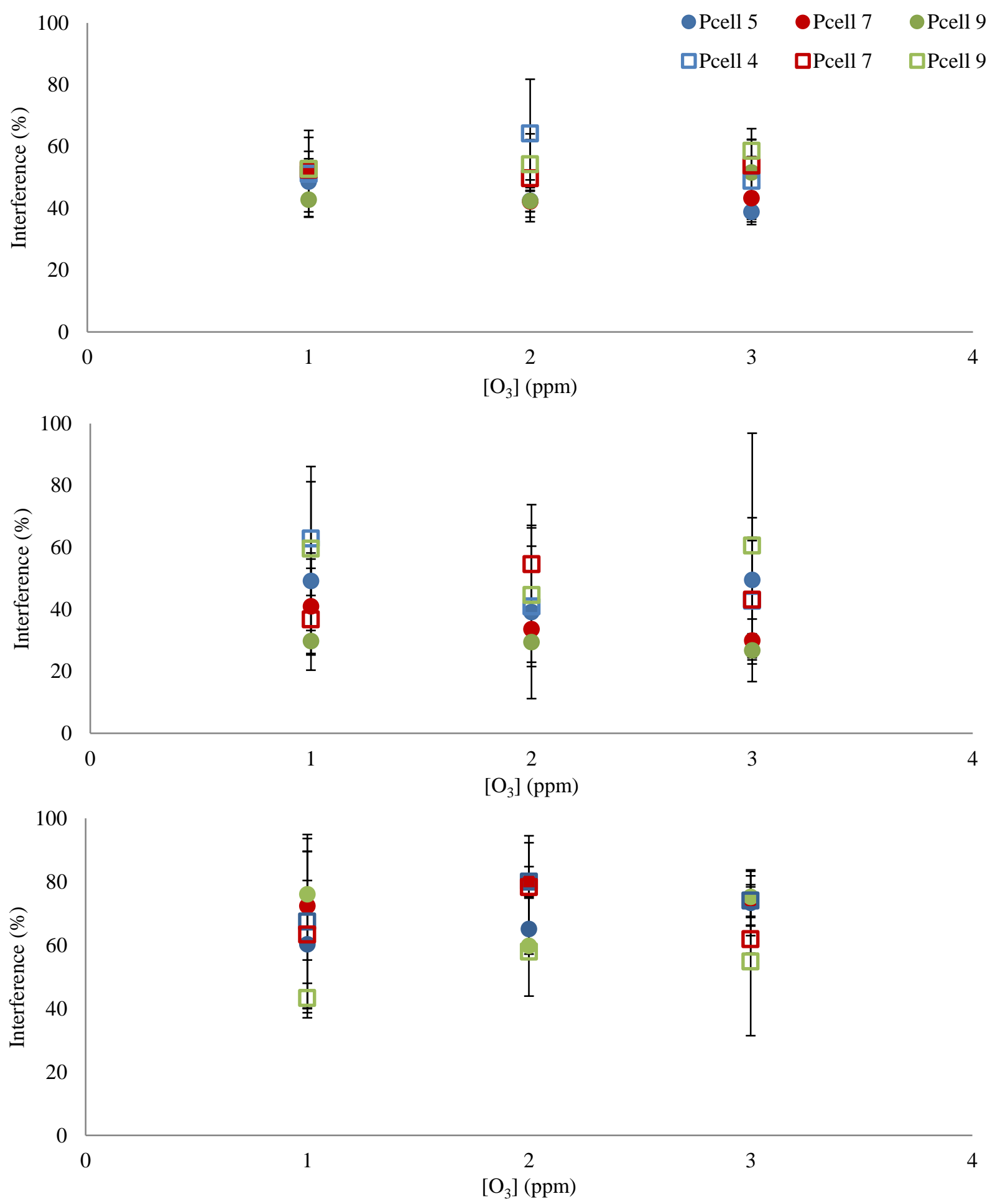

Figure S5. Interference percentages from $\alpha$-pinene ozonolysis at 3 cell pressures (in Torr). Filled circles show measurements using the $1 \mathrm{~mm}$ inlet and the open squares measurement using the $0.6 \mathrm{~mm}$ inlet using the short (top), medium (middle), and long (bottom) inlet lengths. Error bars indicate the precision of the measurement $(2 \sigma)$. 

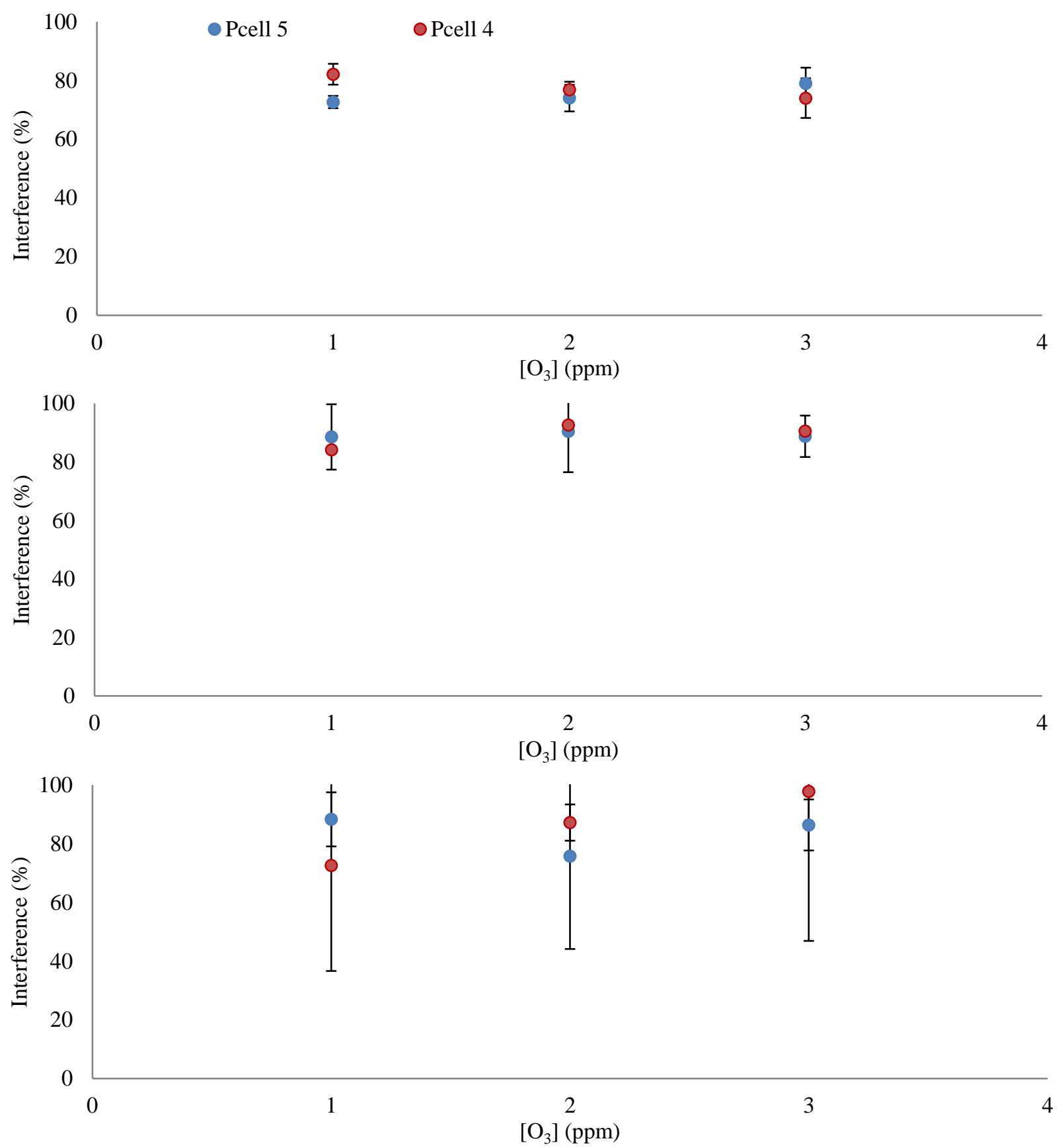

Figure S6. Interference percentages from the ozonolysis of ocimene with an ocimene concentration of approximately $1 \times 10^{13}$ $\mathrm{cm}^{-3}$. The blue circles represent results using the $1 \mathrm{~mm}$ inlet diameter at 5 Torr and the red circles represent results using the 0.6 $\mathrm{mm}$ inlet diameter at 4 Torr. Top plot shows the results for the short inlet, middle plot shows results for the medium inlet, and the bottom plot shows results for the long inlet. Error bars represent the precision of the measurements $(2 \sigma)$. 